\title{
Neutron-neutron correlations in light exotic nuclei $^{1}$
}

\author{
L. Tomio*, M. T. Yamashita ${ }^{\dagger}$ and T. Frederico** \\ *Instituto de Física Teórica, UNESP, 01405-900, São Paulo, SP, Brazil \\ ${ }^{\dagger}$ Laboratório do Acelerador Linear, Instituto de Física, Universidade de São Paulo, CEP \\ 05315-970, São Paulo, Brazil \\ ** Dep. de Física, Instituto Tecnológico de Aeronáutica, Centro Técnico Aeroespacial, 12228-900 \\ São José dos Campos, Brasil
}

\begin{abstract}
For light exotic nuclei modeled as two neutrons $n$ and a core $A$, we report results for the two-neutron correlation functions and also for the mean-square radii, considering a universal scaling function. The results of our calculations for the neutron-neutron correlation functions are qualitatively consistent with recent data obtained for ${ }^{11} \mathrm{Li}$ and ${ }^{14} \mathrm{Be}$ nuclei. The root-mean-square distance in the halo of such nuclei are also consistent with data, which means that the neutrons of the halo have a large probability to be found outside the interaction range. Therefore the lowenergy properties of these halo neutrons are, to a large extend, model independent as long as few physical input scales are fixed. The model is restricted to $s$-wave subsystems, with small energies for the bound or virtual states. For the radii we are also shown results for the ${ }^{6} \mathrm{He}$ and ${ }^{20} \mathrm{C}$. All the interaction effects, as higher partial wave in the interaction and/or Pauli blocking effect are, to some extend, included in our model, as long as the three-body binding energy is supplied.
\end{abstract}

Considering a renormalized three-body model with a pairwise pointlike interaction, we report results obtained for the two-neutron correlation functions and also for the meansquare radii of light exotic nuclei modeled as two neutrons and a core $(n-n-A)$. The results are derived from a universal scaling function that depends on the mass ratio of the neutron and the core, as well as on the nature of the subsystems, bound or virtual. The model consider a minimal number of physical inputs, which are directly related to observables: the two-neutron separation energy $S(2 n)=-E 3$, the neutron-neutron and neutron-core $s$-wave scattering lengths (or the corresponding virtual or bound energies).

The results of our calculations are compared with recent data for the neutron-neutron root-mean-square distances, in case of the halo nuclei ${ }^{6} \mathrm{He},{ }^{11} \mathrm{Li}$ and ${ }^{14} \mathrm{Be}$ nuclei. We also made an estimate prediction for ${ }^{20} \mathrm{C}$ system. For the neutron-neutron correlation function we compare our results for ${ }^{11} \mathrm{Li}$ and ${ }^{14} \mathrm{Be}$ with available experimental data. The neutrons of the halo have a large probability to be found outside the interaction range. Therefore the low-energy properties of these halo neutrons are, to a large extend, model independent as long as few physical input scales are fixed. The model provides a good insight into the three-body structure of halo nuclei, even considering some of its limitations. It is restricted to $s$-wave subsystems, with small energies for the bound or virtual states. We note that, all the interaction effects, such as higher partial wave in the

\footnotetext{
${ }^{1}$ Work supported by the Brazilian agencies FAPESP and CNPq.
} 
TABLE 1. Results of the neutron-neutron root mean-square radii in halo nuclei. Table from Ref. [1]. The virtual states are indicated by (v). The $n n$ virtual state energy was taken as $-143 \mathrm{keV}$.

\begin{tabular}{|c|c|c|c|c|}
\hline Core & $E_{3}(\mathbf{M e V})$ & $E_{n A}(\mathbf{M e V})$ & $\sqrt{\left\langle r_{n n}^{2}\right\rangle}(\mathbf{f m})$ & ${\sqrt{\left\langle r_{n n}^{2}\right\rangle_{e x p}}}_{\text {efm) }}$ \\
\hline \multirow{3}{*}{${ }^{4} \mathrm{He}$} & \multirow{3}{*}{0.973} & 0 & 5.1 & \multirow{3}{*}{$5.9 \pm 1.2$} \\
\hline & & $0.3(\mathrm{v})$ & 4.6 & \\
\hline & & $4.0(\mathrm{v})$ & 3.6 & \\
\hline \multirow{3}{*}{${ }^{9} \mathrm{Li}$} & \multirow{3}{*}{0.29} & 0 & 9.7 & \multirow{3}{*}{$6.6 \pm 1.5$} \\
\hline & & $0.05(\mathrm{v})$ & 8.5 & \\
\hline & & $0.8(\mathrm{v})$ & 6.7 & \\
\hline \multirow[t]{2}{*}{${ }^{12} \mathrm{Be}$} & 1.337 & 0 & 4.6 & \multirow[t]{2}{*}{$5.4 \pm 1.0$} \\
\hline & & $0.2(\mathrm{v})$ & 4.2 & \\
\hline \multirow[t]{2}{*}{${ }^{18} C$} & 3.50 & 0.16 & 3.0 & - \\
\hline & & 0.53 & 4.4 & - \\
\hline
\end{tabular}

interaction and/or Pauli blocking effect are, to some extend, included in the model, as long as the three-body binding energy is supplied.

In order to analyze weakly-bound three-body systems, it is useful a classification scheme as given in [1]. The most natural case is where all the two-body subsystems are bound. We call it all-bound three-body system. In a quite different configuration of three-body system, the case where all the two-body subsystems are unbound, we have the well-known Borromean system. In intermediate situations we can distinguish two other configurations: the Tango case [2], where only one pair of the subsystems is bound; and the Samba [1] case, where only one pair is unbound. Within these configurations, by solving the corresponding Faddeev equations, with renormalized zero-range two-body interactions, we obtain some general properties related to the particle distributions in the halo [1,3]. Our results, for the radii and for the two-neutron correlation functions, can be summarized in Table 1 and in Figs. 1(a) and 1(b).

In Table 1, we present our results for the radii of the three-body halo nuclei ${ }^{6} \mathrm{He}$, ${ }^{11} \mathrm{Li},{ }^{14} \mathrm{Be}$ and ${ }^{20} \mathrm{C}$. A few energies for the (bound/virtual) subsystems are considered according to references given in [1]. When available, the experimental results for the radii are shown in the last column.

In Figs. 1(a) and 1(b) we present results for the neutron-neutron correlation functions, $C_{n n}\left(\vec{p}_{n n}\right)$, in case of the halo-nuclei ${ }^{11} \mathrm{Li}$ and ${ }^{14} \mathrm{Be}$, respectively. The neutron-neutron correlation function, for the $n-n-A$ three-body system, is given by

$$
C_{n n}\left(\vec{p}_{n n}\right)=\frac{\int d^{3} q_{n n}\left|\Psi_{A n n}\left(\vec{q}_{n n}, \vec{p}_{n n}\right)\right|^{2}}{\int d^{3} q_{n n} \int d^{3} q_{n A}\left|\Psi_{A n n}\left(\vec{q}_{n A}, \vec{p}_{n A}\right)\right|^{2} \int d^{3} q_{n^{\prime} A}\left|\Psi_{A n n}\left(\vec{p}_{n^{\prime} A}, \vec{q}_{n^{\prime} A}\right)\right|^{2}},
$$

where $\Psi$ is the corresponding wave function in the Jacobi relative momentum coordinates. With $\gamma=n$ or $A, \vec{p}_{n \gamma}$ is the relative momentum between the particles $n \gamma$, and $\vec{q}_{n \gamma}$ the relative momentum between the expectator particle and the center-of-mass of the pair $n \gamma$.

In conclusion, we observe that our model, with renormalized zero-range two-body interaction, when applied to three-body system with $s$-wave subsystems and with small 

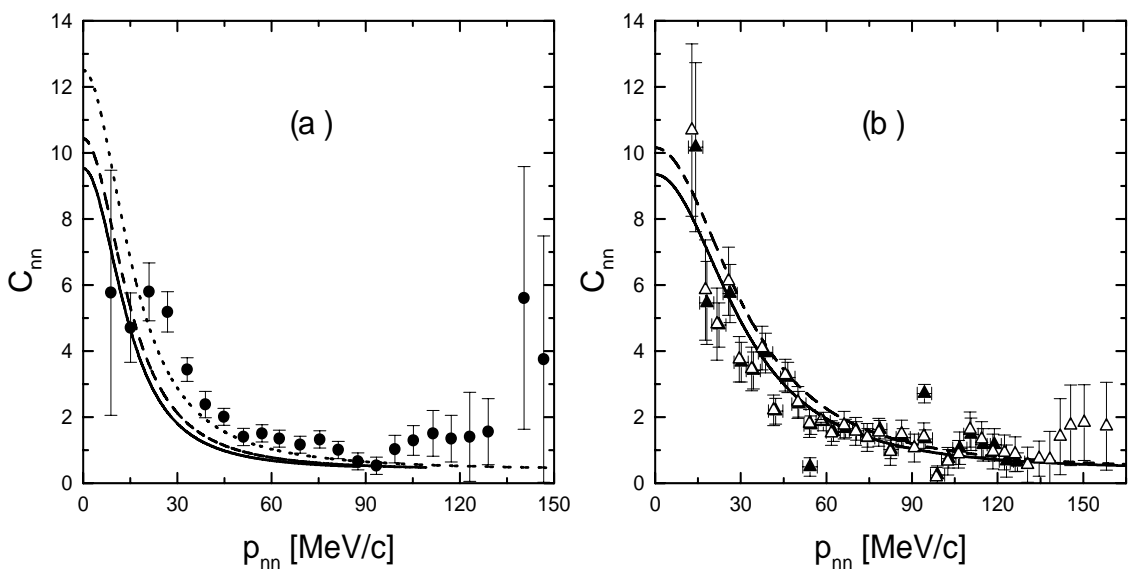

FIGURE 1. Correlation functions for ${ }^{11} \mathrm{Li}$ (a) and for ${ }^{14} \mathrm{Be}(\mathrm{b})$ as functions of the relative momentum, $p_{n n}$, of halo neutrons. Experimental data in (a) are from [4]; and, in (b), from [4] (open triangles) and [5] (solid triangles). The three-body energies was set as $0.29 \mathrm{MeV}$, for ${ }^{11} \mathrm{Li}$; and, $1.337 \mathrm{MeV}$, for ${ }^{14} \mathrm{Be}$. In (a), the solid, dashed and dotted lines are, respectively, the results for $E_{n A}=0,0.05$ and $0.8 \mathrm{MeV}$. In (b), the solid and dashed lines are, respectively, the results for $E_{n A}=0$ and $0.2 \mathrm{MeV}$.

bound or virtual two-body energies, give results consistent with available experimental results. With respect to the size of weakly-bound three-body system $n-n-A$, when we consider all configurations (Borromean, tango, samba and all-bound) with the same three-body energy, we verify the following interesting relation for the two-particle meansquare distances:

$$
\left.\left\langle r^{2}\right\rangle\right|_{\text {Borromean }}<\left.\left\langle r^{2}\right\rangle\right|_{\text {Tango }}<\left.\left\langle r^{2}\right\rangle\right|_{\text {Samba }}<\left.\left\langle r^{2}\right\rangle\right|_{\text {All-bound }} .
$$

Our results for the correlation functions and also for the radii are qualitatively consistent with recent data for the ${ }^{11} \mathrm{Li}$ and ${ }^{14} \mathrm{Be}$ nuclei, which means that the neutrons of the halo have a large probability to be found outside the interaction range. The model provides a good insight into the three-body structure of halo nuclei, even considering some of its limitations: it is restricted to $s$-wave subsystems, with small energies for the bound or virtual states. However, considering that in our model the three-body binding energy is supplied, interaction effects as higher partial waves and/or Pauli blocking effect are, to some extend, included.

\section{REFERENCES}

1. M. T. Yamashita, L. Tomio and T. Frederico, Nucl. Phys. A 735, 40 (2004).

2. F. Robicheaux, Phys. Rev. A 60, 1706 (1999).

3. M. T. Yamashita, R. S. Marques de Carvalho, L. Tomio and T. Frederico, Phys. Rev. A 68, 012506 (2003).

4. F. M. Marqués, et al, Phys. Lett. B 476, 219 (2000).

5. F. M. Marqués, et al, Phys. Rev. C 64, 061301 (2001). 
Copyright of AIP Conference Proceedings is the property of American Institute of Physics and its content may not be copied or emailed to multiple sites or posted to a listserv without the copyright holder's express written permission. However, users may print, download, or email articles for individual use. 